3rd Int'l Conf. on Numerical Analysis and Optimization Sultan Qaboos University, Muscat, Oman, 5-9 Jan. 2014

\title{
The AMPL Modeling Language - an Aid to Formulating and Solving Optimization Problems
}

David M. Gay

AMPL Optimization, Inc.

dmg@ampl.com

http://www .ampl.com 


\section{AMPL summary}

AMPL: a language for mathematical programming problems, e.g.,

$$
\begin{gathered}
\operatorname{minimize} f(x) \\
\text { s.t. } \ell \leq c(x) \leq u,
\end{gathered}
$$

with $x \in \mathbb{R}^{n}$ and $c: \mathbb{R}^{n} \rightarrow \mathbb{R}^{m}$ given algebraically and some $x_{i}$ discrete. 


\section{AMPL goals}

- Easy transcription from math (avoid mistakes)

- Explicit indexing (no hidden magic)

- Declare before use (one-pass reading)

- Separate model, data, commands (orthogonality)

- Separate solvers (open solver interface)

- Update entities as needed (lazy eval.)

- Builtin math. prog. stuff (presolve, red. costs, ...)

- Aim for large scale nonlinear (sparsity, generality) 


\section{AMPL history}

- Early 1980's: little languages at Bell Labs

- 1983: Fourer's TOMS paper, ML's vs MG's

- 1984: Karmarkar hoopla begins

- 1985-6: Fourer sabbatical at Bell Labs

- 1987: AMPL CSTR (Fourer, Gay, Kernighan)

- Late 1980's: reimplementation; extensions begin

- 1993: 1st edition of AMPL book

- 2001: collapse of Bell Labs

- 2003: 2nd edition of AMPL book; dmg $\rightarrow$ Sandia

- 2010: dmg $\rightarrow$ AMPL Optimization 


\section{Simple declarations, commands}

$$
\begin{aligned}
& \text { ampl: param } \mathrm{p} ; \\
& \text { ampl: param } \mathrm{q}=\mathrm{p}+10 ; \\
& \text { ampl: data; param } \mathrm{p}:=2.5 ; \\
& \text { ampl data: display } \mathrm{p}, \mathrm{q} ; \\
& \mathrm{p}=2.5 \\
& \mathrm{q}=12.5 \\
& \text { ampl: let } \mathrm{p}:=17 \text {; display } \mathrm{p}, \mathrm{q} ; \\
& \mathrm{p}=17 \\
& \mathrm{q}=27
\end{aligned}
$$




\section{Simple sets}

ampl: set $A$; set $B$;

ampl: $\operatorname{set} C=p \ldots q$;

ampl: display A;

Error executing "display" command:

no data for set $A$

ampl: data; set $A:=a b c$; set $B:=c d$; ampl data: display A, B, C;

set $A:=a b c$;

set $B:=c d$;

set $\mathrm{C}:=\begin{array}{lllllllllll}17 & 18 & 19 & 20 & 21 & 22 & 23 & 24 & 25 & 26 & 27\end{array}$ 


\section{Simple set operations}

ampl: display $A$ intersect $B, A$ union $B$; set $A$ inter $B:=c$;

set $A$ union $B:=a b c d$;

ampl: display A diff $B, A$ symdiff $B$;

set $A \operatorname{diff} B:=a b$;

set $A$ symdiff $B:=a b d$; 


\section{Iterated expressions, declarations}

ampl: print sum $\{i$ in $1 . .4\}$;

10

ampl: print prod $\{i$ in $1 \ldots 4\}$;

24

ampl: param fac $\{i$ in $1 . .9\}$

ampl? = if $i==1$ then 1 else $i * f a c[i-1]$;

ampl: print $\max \{i$ in $1 . .9\}$

ampl? $\operatorname{abs}(f a c[i]-\operatorname{prod}\{j$ in $2 . . i\} j)$;

0 


\section{More iterated commands}

ampl: display fac, $\{i$ in $1 \ldots 9\} \operatorname{prod}\{j$ in $2 \ldots i\} j$; : fac $\operatorname{prod}\{j$ in $2 \ldots i\} j:=$

1

1

1

2

2

2

3

6

6

4

24

24

5120

120

6

720

720

7

5040

5040

840320

40320

9362880

362880 


\section{Example model: diet.mod}

set NUTR; set FOOD;

param cost $\{$ FOOD $\}>0$;

param f_min $\{$ FOOD $\}>=0$;

param f_max $\{j$ in FOOD $\}>=f_{-} \min [j]$;

param n_min $\{$ NUTR $\}>=0$;

param n_max $\{i$ in NUTR $\}>=n_{-} \min [i]$;

param amt $\{$ NUTR, FOOD $\}>=0$;

$\operatorname{var}$ Buy $\{j$ in FOOD $\}>=f_{-} \min [j],<=f_{-} \max [j]$;

minimize Total_Cost:

sum $\{j$ in FOOD $\}$ cost $[j] *$ Buy $[j]$;

subject to $D$ iet $\{i$ in NUTR $\}$ :

$$
\begin{aligned}
n_{-} \min [i] & <=\operatorname{sum}\{j \text { in FOOD }\} \text { amt }[i, j] * \operatorname{Buy}[j] \\
& <=n_{-} \max [i]
\end{aligned}
$$




\section{Example data file: diet.dat (beginning)}

data;

set NUTR :=A B1 B2 C ;

set FOOD $:=$ BEEF CHK FISH HAM MCH MTL SPG TUR ;

param: cost f_min f_max :=

$\begin{array}{llll}\text { BEEF } & 3.19 & 0 & 100 \\ \text { CHK } & 2.59 & 0 & 100 \\ \text { FISH } & 2.29 & 0 & 100 \\ \text { HAM } & 2.89 & 0 & 100 \\ \text { MCH } & 1.89 & 0 & 100 \\ \text { MTL } & 1.99 & 0 & 100 \\ \text { SPG } & 1.99 & 0 & 100 \\ \text { TUR } & 2.49 & 0 & 100 ;\end{array}$




\section{Example data file continued: diet.dat}

$\begin{array}{ccc}\text { param: } & \text { n_min } & \text { n_max : } \\ \text { A } & 700 & 10000 \\ \text { C } & 700 & 10000 \\ \text { B1 } & 700 & 10000 \\ \text { B2 } & 700 & 10000 ;\end{array}$

param amt (tr):

A C B1 B2 :=

$\begin{array}{lllll}\text { BEEF } & 60 & 20 & 10 & 15\end{array}$

$\begin{array}{lllll}\text { CHK } & 8 & 0 & 20 & 20\end{array}$

$\begin{array}{lllll}\text { FISH } & 8 & 10 & 15 & 10\end{array}$

$\begin{array}{lllll}\text { HAM } & 40 & 40 & 35 & 10\end{array}$

$\begin{array}{lllll}\mathrm{MCH} & 15 & 35 & 15 & 15\end{array}$

$\begin{array}{lllll}\text { MTL } & 70 & 30 & 15 & 15\end{array}$

$\begin{array}{lllll}\text { SPG } & 25 & 50 & 25 & 15\end{array}$

$\begin{array}{lllll}\text { TUR } & 60 & 20 & 15 & 10 \text {; }\end{array}$ 


\section{Example session}

ampl: model diet.mod; data diet.dat;

ampl: solve;

MINOS 5.51: optimal solution found.

6 iterations, objective 88.2

ampl: display Buy;

Buy $[*]:=$

BEEF 0

CHK 0

FISH 0

HAM 0

MCH $\quad 46.6667$

MTL $1.57618 \mathrm{e}-15$

SPG $8.42982 \mathrm{e}-15$

TUR 0 


\section{Example session continued:}

\section{Imposing integrality}

ampl: redeclare var $\operatorname{Buy}\{j$ in FOOD $\}$

ampl? integer $>=f \_m i n[j]<=f_{-} \max [j]$;

ampl: solve;

MINOS 5.51: ignoring integrality of 8 variables

MINOS 5.51: optimal solution found.

4 iterations, objective 88.2

ampl: option solver cplex;

ampl: solve;

CPLEX 12.6.0.0: optimal integer solution; objective 88.44

4 MIP simplex iterations

0 branch-and-bound nodes 


\section{Example session continued: result of imposing integrality}

\begin{tabular}{lr}
\multicolumn{2}{l}{ ampl: disp } \\
Buy [*] : \\
BEEF & 0 \\
CHK & 2 \\
FISH & 0 \\
HAM & 0 \\
MCH & 43 \\
MTL & 1 \\
SPG & 0 \\
TUR & 0 \\
; &
\end{tabular}




\section{Example: modified data (diet2.dat):}

data;

set NUTR := A B1 B2 C NA CAL ;

set FOOD $:=$ BEEF CHK FISH HAM MCH MTL SPG TUR ;

$\begin{array}{cccc}\text { param: } & \text { cost } & \text { f_min } & \text { f_max }:= \\ \text { BEEF } & 3.19 & 2 & 10 \\ \text { CHK } & 2.59 & 2 & 10 \\ \text { FISH } & 2.29 & 2 & 10 \\ \text { HAM } & 2.89 & 2 & 10 \\ \text { MCH } & 1.89 & 2 & 10 \\ \text { MTL } & 1.99 & 2 & 10 \\ \text { SPG } & 1.99 & 2 & 10 \\ \text { TUR } & 2.49 & 2 & 10 ;\end{array}$




\section{Example: more diet2.dat:}

$\begin{array}{ccc}\text { param: } & \text { n_min } & \text { n_max := } \\ \text { A } & 700 & 20000 \\ \text { C } & 700 & 20000 \\ \text { B1 } & 700 & 20000 \\ \text { B2 } & 700 & 20000 \\ \text { NA } & 0 & 40000 \\ \text { CAL } & 16000 & 24000 ;\end{array}$

param amt (tr):

A $\mathrm{C} \quad \mathrm{B} 1 \quad \mathrm{~B} 2 \quad \mathrm{NA} \quad \mathrm{CAL}:=$

$\begin{array}{lllllll}\mathrm{BEEF} & 60 & 20 & 10 & 15 & 938 & 295\end{array}$

$\begin{array}{lllllll}\text { CHK } & 8 & 0 & 20 & 20 & 2180 & 770\end{array}$

$\begin{array}{lllllll}\text { FISH } & 8 & 10 & 15 & 10 & 945 & 440\end{array}$

$\begin{array}{lllllll}\text { HAM } & 40 & 40 & 35 & 10 & 278 & 430\end{array}$

$\begin{array}{lllllll}\text { MCH } & 15 & 35 & 15 & 15 & 1182 & 315\end{array}$

$\begin{array}{lllllll}\text { MTL } & 70 & 30 & 15 & 15 & 896 & 400\end{array}$

$\begin{array}{lllllll}\text { SPG } & 25 & 50 & 25 & 15 & 1329 & 370\end{array}$

$\begin{array}{llllllll}\text { TUR } & 60 & 20 & 15 & 10 & 1397 & 450 & \text {; }\end{array}$ 


\section{Using the new data file}

ampl: reset data;

ampl: data diet2.dat;

ampl: solve;

CPLEX 12.6.0.0: integer infeasible.

1 MIP simplex iterations

0 branch-and-bound nodes

No basis.

ampl: display Buy;

Buy $[*]:=$

BEEF 0

CHK 0

FISH 0

HAM 0

$\mathrm{MCH} \quad \mathrm{O}$

MTL 0

SPG 0

TUR 0 


\section{Analyzing the infeasibility}

ampl: option solver minos; solve;

MINOS 5.51: ignoring integrality of 8 variables MINOS 5.51: infeasible problem.

9 iterations ampl: display diet.lb, diet.body, diet.ub, diet.slack; : diet.lb diet.body diet.ub diet.slack := $\begin{array}{lllll}\text { A } & 700 & 1993.09 & 20000 & 1293.09\end{array}$

$\begin{array}{lllll}\text { B1 } & 700 & 841.091 & 20000 & 141.091\end{array}$

$\begin{array}{lllll}\text { B2 } & 700 & 601.091 & 20000 & -98.9086\end{array}$

$\begin{array}{lllll}\text { C } & 700 & 1272.55 & 20000 & 572.547\end{array}$

$\begin{array}{lllll}\text { CAL } & 16000 & 17222.9 & 24000 & 1222.92\end{array}$

$\mathrm{NA}$

$040000 \quad 40000$

$7.27596 e-12$ 


\section{Fixing the infeasibility}

ampl: print $\mathrm{n}_{-} \max \left[{ }^{\prime} \mathrm{NA}{ }^{\prime}\right]$;

40000

ampl: let $n_{-} \max \left[{ }^{\prime} N A '\right]:=50000$;

ampl: solve;

MINOS 5.51: ignoring integrality of 8 variables

MINOS 5.51: optimal solution found.

5 iterations, objective 118.0594032

ampl: param MinosSoln\{FOOD\};

ampl: let $\{i$ in FOOD $\}$ MinosSoln[i] := Buy[i];

ampl: option solver cplex; solve;

CPLEX 12.6.0.0: optimal integer solution; objective 119.3

9 MIP simplex iterations

0 branch-and-bound nodes

absmipgap $=2.84217 \mathrm{e}-14$, relmipgap $=2.38237 \mathrm{e}-16$ 


\section{Looking at the solutions}

$\begin{array}{lrc}\text { ampl : display Buy, Mi } & \text { dinos. } \\ : & \text { Buy } & \text { Minoln } \\ \text { BEEF } & 9 & 5.36061 \\ \text { CHK } & 2 & 2 \\ \text { FISH } & 2 & 2 \\ \text { HAM } & 8 & 10 \\ \text { MCH } & 10 & 10 \\ \text { MTL } & 10 & 10 \\ \text { SPG } & 7 & 9.30605 \\ \text { TUR } & 2 & 2 \\ \text {; } & & \end{array}$




\section{A nonlinear example, pgon.mod}

\# Maximum area for unit-diameter polygon of $\mathrm{N}$ sides.

\# The following model started as a GAMS model by Francisco J. Prieto.

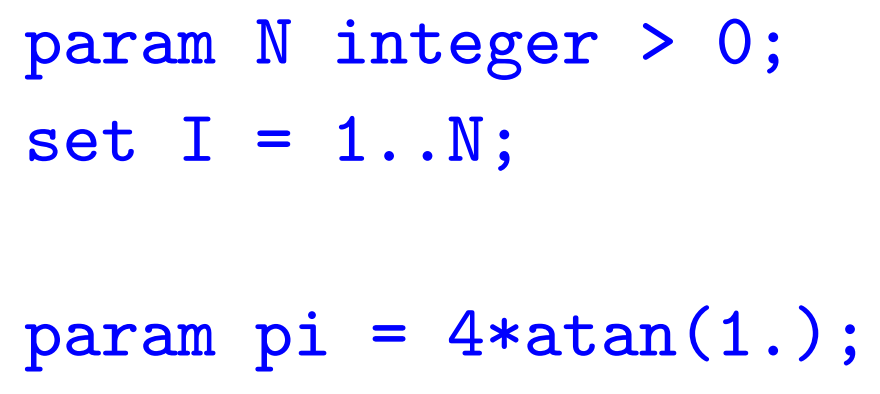

s.t. $\operatorname{cd}\{i$ in $I, j$ in $i+1 \ldots N\}$ :

$$
\text { rho[i] } * * 2+\operatorname{rho}[j] * * 2
$$

- $2 * \operatorname{rho}[i] * \operatorname{rho}[j] * \cos (\operatorname{theta}[j]-\operatorname{theta}[i])<=1$; 


\section{A nonlinear example (con'd), pgon.mod}

s.t. $\operatorname{ac}\{i$ in $2 . . N\}$ :

theta $[i]>=$ theta $[i-1]$;

s.t. fix_theta: theta[N] = pi;

s.t. fix_rho: $\quad r h o[N]=0$;

maximize area:

$.5 * \operatorname{sum}\{i$ in $2 \ldots N\}$ rho[i]*rho[i-1]*sin(theta[i] - theta[i-1]); 


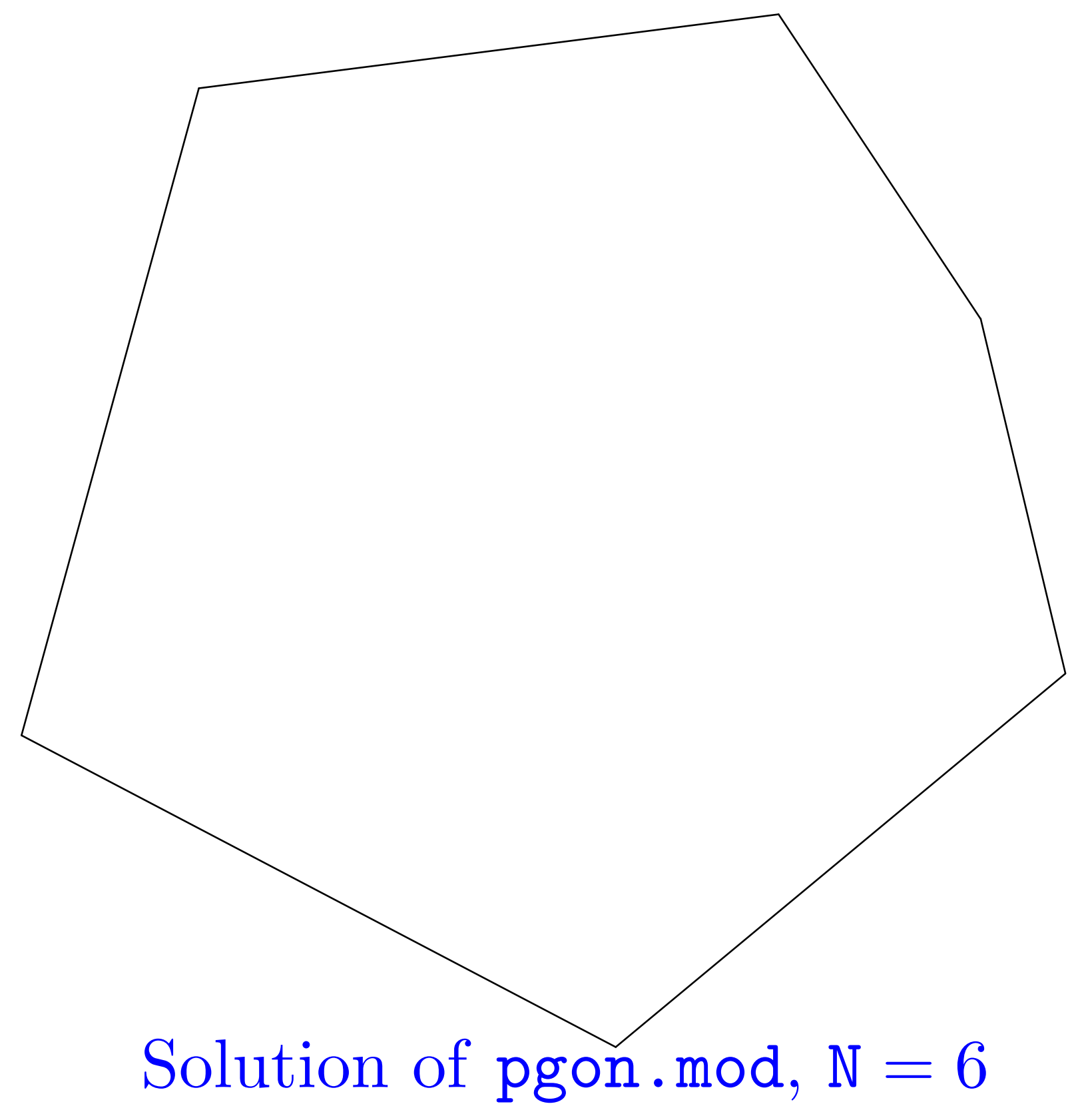




\section{Solution does not lie on a circle}

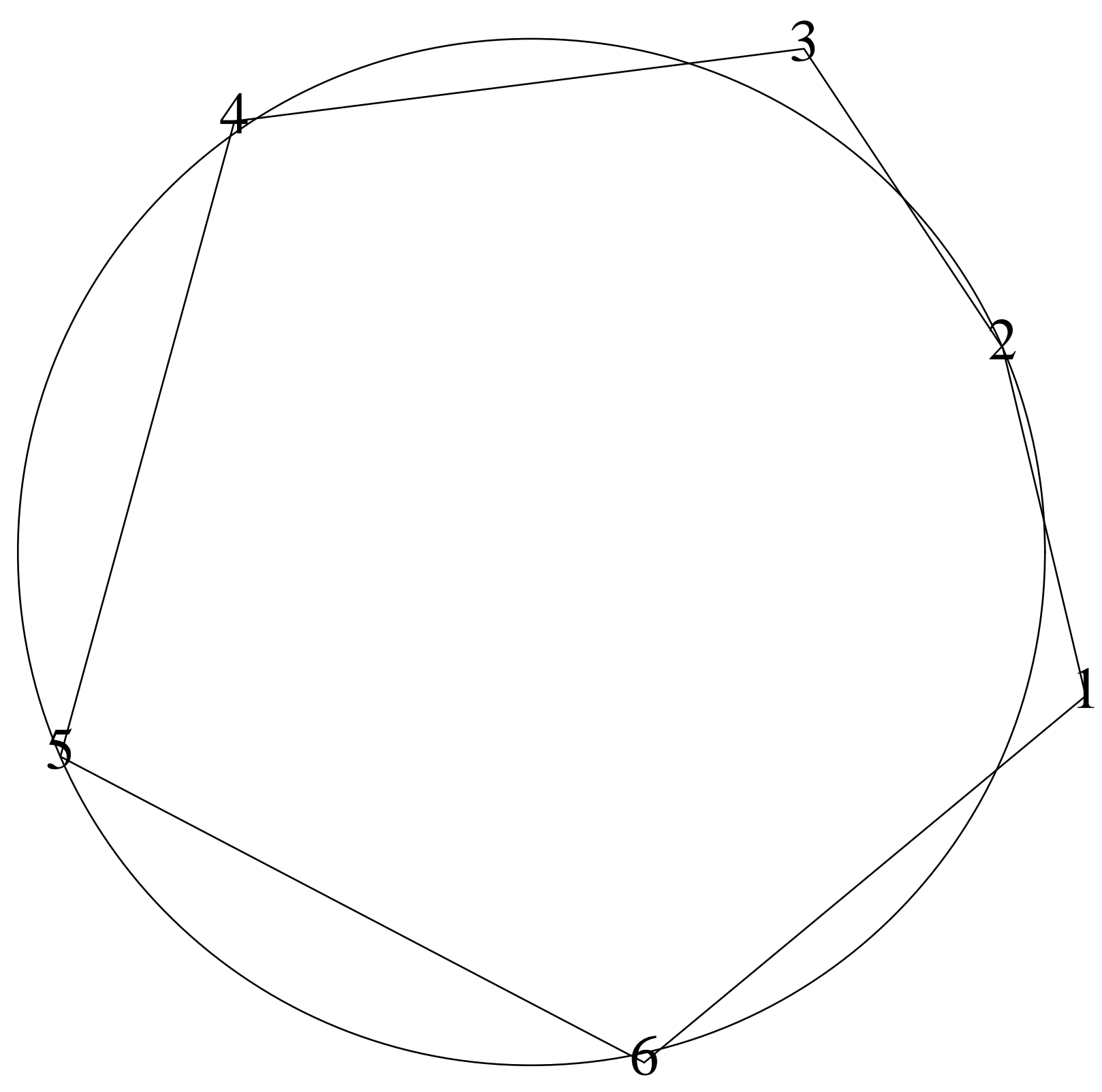


Commands in file "pgwrite" used via

$$
\text { include pgwrite }
$$

Or

$$
\text { commands pgwrite; }
$$

printf ".PS 6i\nline from 0,0" >pgon60.ms; printf $\{i$ in $I\}$ " $\backslash \backslash \backslash n \backslash$ tto $\% g, \% g "$, rho $[i] * \cos ($ theta $[i])$, rho[i]*sin(theta[i]) >ppon60.ms; printf "; \n.PE\n" >>pgon60.ms; close pgon.ms; 


\section{Slices can turn $O\left(n^{2}\right)$ into $O(n)$}

Example where changing

s.t. $c\{a$ in $A\}$ :

$\operatorname{sum}\{(i, j)$ in $S: i==a\} \quad x[i, j]==1$;

into

s.t. $c\{a$ in $A\}$ :

$\operatorname{sum}\{(a, j)$ in $S\} \times[a, j]==1$;

reduced problem instantiation from 4 hours to a minute. 


\section{Iterated union via setof}

\#\# Portion of a data-fusion model

set A dimen 2;

param $\mathrm{E}\{\mathrm{A}\}$;

set $I=\operatorname{setof}\{(i, j)$ in $A\} i ; \#$ observations

set $J=\operatorname{setof}\{(i, j)$ in $A\} j ; \#$ classifiers

param $y\{I\}$ in $\{1,-1\}$;

$\operatorname{var} \mathrm{x}\{\mathrm{J}\}>=0$;

set $B=\{(i, j)$ in $A: y[i] * E[i, j]<0\}$; \# mis-classified pairs

minimize errsq: $\operatorname{sum}\{i$ in $I\}(\operatorname{sum}\{(i, j)$ in $B\}$ y[i]*E[i,j]*x[j])^2;

s.t. convex: $\operatorname{sum}\{i$ in $J\} x[i]=1$;

param Majority $=$ floor $(\operatorname{card}(\mathrm{J}) / 2)+1$;

\# bad training cases with simple voting

set Badvote $=\{i$ in $I: \operatorname{card}\{(i, j)$ in $B\}>=$ Majority $\}$; 


\section{Iterated union, defined var}

\# Mesh untangling constraints, right-hand rule at each vertex. set $\mathrm{D} 3=1 \ldots 3$;

\# three spatial coordinates

param Npoints;

set $P$ default $0 \ldots$ Npoints-1; \# set of points

$\operatorname{var} \mathrm{V}\{\mathrm{P}, \mathrm{D} 3\}$;

\# vertices

param Nfixed default 0 ;

set Fixed within $P$ default $\operatorname{card}(P)-N$ fixed $\ldots \operatorname{card}(P)-1$;

set Hexes within $\{P, P, P, P, P, P, P, P\}$;

set Edges = union $\{(\mathrm{a}, \mathrm{b}, \mathrm{c}, \mathrm{d}, \mathrm{e}, \mathrm{f}, \mathrm{g}, \mathrm{h})$ in Hexes $\}\{$

$$
\begin{aligned}
& (a, b),(a, d),(a, e), \\
& (b, c),(b, a),(b, f), \\
& (c, d),(c, b),(c, g), \\
& (d, a),(d, c),(d, h), \\
& (e, h),(e, f),(e, a), \\
& (f, e),(f, g),(f, b), \\
& (g, f),(g, h),(g, c), \\
& (h, g),(h, e),(h, d)\} ;
\end{aligned}
$$

var $\operatorname{dx}\{(a, b)$ in Edges, $j$ in $D 3\}=v[b, j]-v[a, j]$; 


\section{Iterated union, defined var (con'd)}

set HexCorners $=\{(a, b, c, d, e, f, g, h)$ in Hexes,

$$
\begin{aligned}
(A, B, D, E) \text { in }\{ & (a, b, d, e), \\
& (b, c, a, f), \\
& (c, d, b, g), \\
& (d, a, c, h), \\
& (e, h, f, a), \\
& (f, e, g, b), \\
& (g, f, h, c), \\
& (h, g, e, d)\}\} ;
\end{aligned}
$$

$\operatorname{var} \operatorname{volsign}\{(a, b, c, d, e, f, g, h, A, B, D, E)$ in HexCorners $\}=$

$$
\begin{aligned}
& \mathrm{dx}[\mathrm{A}, \mathrm{B}, 1] *(\mathrm{dx}[\mathrm{A}, \mathrm{D}, 2] * \mathrm{dx}[\mathrm{A}, \mathrm{E}, 3]-\mathrm{dx}[\mathrm{A}, \mathrm{D}, 3] * \mathrm{dx}[\mathrm{A}, \mathrm{E}, 2])+ \\
& \mathrm{dx}[\mathrm{A}, \mathrm{B}, 2] *(\mathrm{dx}[\mathrm{A}, \mathrm{D}, 3] * \mathrm{dx}[\mathrm{A}, \mathrm{E}, 1]-\mathrm{dx}[\mathrm{A}, \mathrm{D}, 1] * \mathrm{dx}[\mathrm{A}, \mathrm{E}, 3])+ \\
& \mathrm{dx}[\mathrm{A}, \mathrm{B}, 3] *(\mathrm{dx}[\mathrm{A}, \mathrm{D}, 1] * \mathrm{dx}[\mathrm{A}, \mathrm{E}, 2]-\mathrm{dx}[\mathrm{A}, \mathrm{D}, 2] * \mathrm{dx}[\mathrm{A}, \mathrm{E}, 1]) ;
\end{aligned}
$$

var $\mathrm{mn}$; maximize maximin: $\mathrm{mn}$;

s.t. $m n \_b o u n d ~\{(a, b, c, d, e, f, g, h, A, B, D, E)$ in HexCorners $\}$ :

$$
\mathrm{mn}<=\text { volsign }[\mathrm{a}, \mathrm{b}, \mathrm{c}, \mathrm{d}, \mathrm{e}, \mathrm{f}, \mathrm{g}, \mathrm{h}, \mathrm{A}, \mathrm{B}, \mathrm{D}, \mathrm{E}] \text {; }
$$

s.t. Volsign $\{(a, b, c, d, e, f, g, h, A, B, D, E)$ in HexCorners $\}$ :

$$
\text { volsign }[a, b, c, d, e, f, g, h, A, B, D, E]>=0 \text {; }
$$




\section{AMPL flexibility goals}

- Allow interactive, "batch", and "GUI" use

- Allow extensions via shared libs

$\circ$ imported functions

- table handlers

- Promote interaction with host OS

o shell command

$\circ$ pipe functions

○ options $\longrightarrow$ environment

○ file redirections, remove command 


\section{Options}

Environment: (name, \$value) pairs.

Initial environment from invocation

with defaults for names not there.

Changed by option command.

AMPL interprets some option settings (e.g., \$solver).

Invoked processes (solvers, shell) see modified env.

Convention: option solver_options affects solver. 


\section{Option examples}

option cplex_options 'advance=2 lpdisplay=1 | prestats $=11$

primalopt'

" aggregate=1 aggfill=20";

option solver knitro,

knitro_options "maxit=30"; 


\section{Interactive option examples}

ampl:option cplex_options 'advance=2 lpdisplay=1 ।

prestats $=1 \backslash$

primalopt'

" aggregate=1 aggfill=20" ;

ampl:option cplex_options;

option cplex_options 'advance=2 lpdisplay=1 ।

primalopt aggregate=1 aggfill=20';

ampl:option;

option AMPLFUNC ampltabl.dll;

option Cautions 1;

option MD_precision 0;

option OPTIONS_IN ', ;

option OPTIONS_INOUT ', ;

option OPTIONS_OUT ', ;

option PATH ':/home/dmg/h/bin:/usr/local/bin:/usr/bin:/bin';

option SHELL '/bin/bash'; 


\section{Interactive option examples (con'd)}

ampl:option solver cplex, re*es 1, send_st* 0;

option reset_initial_guesses 1 ;

option send_statuses 0 ;

ampl : solve;

CPLEX 12.6.0.0: advance $=2$

lpdisplay $=1$

primalopt

aggregate $=1$

aggfill $=20$

LP Presolve eliminated 6 rows and 9 columns.

All rows and columns eliminated.

CPLEX 12.6.0.0: optimal integer solution; objective 119.3

9 MIP simplex iterations

0 branch-and-bound nodes

absmipgap $=2.84217 \mathrm{e}-14$, relmipgap $=2.38237 \mathrm{e}-16$ 


\section{Redirections}

Can redirect most output to files:

ampl:option >foo1;

ampl:solve >solve.out;

CPLEX 12.6.0.0: optimal integer solution; objective 119.3

9 MIP simplex iterations

0 branch-and-bound nodes

absmipgap $=2.84217 \mathrm{e}-14$, relmipgap $=2.38237 \mathrm{e}-16$

ampl:close solve.out; shell 'cat solve.out'; 


\section{String expressions}

( string experssion ) can replace 'literal string' almost everywhere.

ampl:param mypath symbolic; ampl:let mypath := 'c:/full/path/to/somewhere/'; ampl:option solver (mypath \& 'minos' \& $5+.4$ ); ampl:option solver; option solver 'c:/full/path/to/somewhere/minos5.4'; ampl:print \$solver; c:/full/path/to/somewhere/minos5.4 


\section{Interaction with Solvers}

AMPL writes .nl file with

- problem statistics

- coefficients for linear expressions

- expression graphs for nonlinear expressions

- initial guesses (primal and dual)

- suffixes (user declared; basis)

AMPL options modify the solver's environment. Solver writes .sol file with solution and returned suffixes (e.g., basis). 


\section{Problem Transformations}

AMPL's presolve derives and propagates bounds with directed roundings and may fix variables, remove constraints (e.g., inequalities that are never tight), resolve complementarities, turn nonlinear expressions into linear (after fixing relevant variables), simplify convex piecewise-linear expressions, and convert nonconvex piecewise-linear expressions to equivalent systems of integer variables and SOS-2 constraints. 


\section{Implementation Techniques}

- lex and yacc; cfront

- expression graph manipulations

- hashing for symbols, sets, common expressions

- lifting invariants out of loops

- on-the-fly expression rewrites

- error handling with registered clean-up routines and longjump

- reference counting where appropriate

- sparse matrix methods 


\section{Hoped-for Enhancements}

- programming interfaces

- AMPL functions for modeling and solver call-backs

- ordered sets of tuples

- tuples as atoms

- more efficient instantiation of related instances (e.g., when adding cutting planes)

- variables in subscripts for constraint programming

- with one objective, exploiting duality in presolve

- extend AMPL/solver-interface library (ASL) for stochastic programming 


\section{More Wish-List Items}

- facilities for SDP and multi-level optimization

- conversations with solvers: just supply instance differences when the next problem instance is not too different from the current one

- units

- other data types (rational, complex)

- parallel ASL evaluations

- constructs for parallelism in AMPL 


\section{AMPL facilities not treated in these slides}

- drop, restore, fix, unfix; named problems

- looping and flow-of-control commands

- suffixes

- tables

- column-generation syntax (e.g., node and arc)

- complementarity constraints

- subscripted sets versus tuples

- imported functions (with OUT-args)

- constraint-programming extensions (partly done) 
AMPL For more details (info@ampl.org or dmg@ampl.com)

http://www.ampl.com points to

- The AMPL book (PDF files now freely available)

- examples (models, data)

- descriptions of new stuff, e.g., new IDE

- Try AMPL! and NEOS; trial licenses

- downloads

- student binaries and requests for course licenses

- solver interface library source

- "standard" table handler \& source

$\circ$ papers and reports 


\section{Selected References}

Robert Fourer, David M. Gay and Brian W. Kernighan, "A Modeling Language for Mathematical Programming", Management Science 36 (1990), pp. 519-554.

Robert Fourer, David M. Gay and Brian W. Kernighan, AMPL: A Modeling Language for Mathematical Programming, Duxbury Press / Brooks/Cole Publishing Company, 2002 (Second edition, ISBN 0-534-38809-4). Individual chapters are now freely avilable for download: see http://www.ampl.com/BOOK/download.html.

David M. Gay, "Automatic Differentiation of Nonlinear AMPL Models", in Automatic Differentiation of Algorithms: Theory, Implementation, and Application, A. Griewank and G. Corliss, eds., SIAM (Philadelphia, 1991), pp. 61-73.

Robert Fourer and David M. Gay, "Experience with a Primal Presolve Algorithm", in Large Scale Optimization: State of the Art, W.W. Hager, D.W. Hearn and P.M. Pardalos, eds., Kluwer Academic Publishers (Dordrecht, 1994), pp. 135-154.

Robert Fourer and David M. Gay, "Expressing Special Structures in an Algebraic Modeling Language for Mathematical Programming", ORSA Journal on Computing 7 (1995), pp. 166-190.

David M. Gay, "More AD of Nonlinear AMPL Models: Computing Hessian Information and Exploiting Partial Separability", in Computational Differentiation: Techniques, Applications, and Tools, M. Berz, C. Bischof, G. Corliss and A. Griewank, eds., SIAM (Philadelphia, 1996), pp. 173-184. 


\section{Seleted References (continued)}

David M. Gay, "Hooking Your Solver to AMPL", Technical report, Bell

Laboratories, Murray Hill, NJ (1993; revised 1994, 1997).

See http://www.ampl.com/REFS/abstracts.html\#hooking2.

Robert Fourer, "Extending a General-Purpose Algebraic Modeling Language to Combinatorial Optimization: A Logic Programming Approach", in Advances in Computational and Stochastic Optimization, Logic Programming, and Heuristic Search: Interfaces in Computer Science and Operations Research, D.L. Woodruff, ed., Kluwer Academic Publishers (Dordrecht, The Netherlands, 1998), pp. 31-74.

Michael C. Ferris, Robert Fourer and David M. Gay, "Expressing Complementarity Problems in an Algebraic Modeling Language and Communicating Them to Solvers", SIAM Journal on Optimization 9 (1999), pp. 991-1009.

Robert Fourer and David M. Gay, "Extending an Algebraic Modeling Language to Support Constraint Programming", INFORMS Journal on Computing 14 \#4 (2002), pp. 322-344. 


\section{Seleted References (continued)}

Robert Fourer and David M. Gay, "Numerical Issues and Influences in the Design of Algebraic Modeling Languages for Optimization", in Proceedings of the 20th Biennial Conference on Numerical Analysis, Dundee, Scotland, D.F. Griffiths and D.A. Watson, eds. Numerical Analysis Report NA/217 (2003), pp. 39-51.

Elizabeth D. Dolan, Robert Fourer, Jean-Pierre Goux, Todd S. Munson and Jason Sarich, "Kestrel: An Interface from Optimization Modeling Systems to the NEOS Server", INFORMS Journal on Computing 20 (2008), pp. 525-538.

David M. Gay, "Bounds from Slopes", report SAND2010-1794P; http://www.sandia.gov/ dmgay/bounds10.pdf.

Christian Kirches and Sven Leyffer, "TACO: a Toolkit for AMPL Control Optimization", Mathematical Programming Computation 5 \#3 (2013). 


\section{These Slides}

PDF file for these slides: click Calendar in http://www . ampl.com to find a pointer to http://www . ampl . com/MEETINGS/ TALKS/2014_01_Muscat_Wed.pdf 